\title{
Impact of Obesity and Hyperglycemia on Placental Mitochondria
}

\author{
Chiara Mandò $\mathbb{D},{ }^{1}$ Gaia Maria Anelli, ${ }^{1}$ Chiara Novielli $\left(\mathbb{D},{ }^{1}\right.$ Paola Panina-Bordignon $\left(\mathbb{D},{ }^{2}\right.$ \\ Maddalena Massari $\mathbb{D}^{1}{ }^{1}$ Martina Ilaria Mazzocco ${ }^{(D},{ }^{1}$ and Irene Cetin ${ }^{1}$ \\ ${ }^{1}$ Department of Biomedical and Clinical Sciences, Unit of Obstetrics and Gynecology, ASST Fatebenefratelli Sacco \\ University Hospital, Università degli Studi di Milano, Via G. B. Grassi 74, 20157 Milano, Italy \\ ${ }^{2}$ Division of Genetics and Cell Biology, IRCCS Ospedale San Raffaele, Via Olgettina 60, 20132 Milano, Italy
}

Correspondence should be addressed to Chiara Mandò; chiara.mando@unimi.it

Received 9 February 2018; Revised 13 June 2018; Accepted 12 July 2018; Published 14 August 2018

Academic Editor: Victor M. Victor

Copyright (C) 2018 Chiara Mandò et al. This is an open access article distributed under the Creative Commons Attribution License, which permits unrestricted use, distribution, and reproduction in any medium, provided the original work is properly cited.

A lipotoxic placental environment is recognized in maternal obesity, with increased inflammation and oxidative stress. These changes might alter mitochondrial function, with excessive production of reactive oxygen species, in a vicious cycle leading to placental dysfunction and impaired pregnancy outcomes. Here, we hypothesize that maternal pregestational body mass index (BMI) and glycemic levels can alter placental mitochondria. We measured mitochondrial DNA (mtDNA, real-time PCR) and morphology (electron microscopy) in placentas of forty-seven singleton pregnancies at elective cesarean section. Thirty-seven women were normoglycemic: twenty-one normal-weight women, NW, and sixteen obese women, OB/GDM(-). Ten obese women had gestational diabetes mellitus, $\mathrm{OB} / \mathrm{GDM}(+) . \mathrm{OB} / \mathrm{GDM}(-)$ presented higher mtDNA levels versus NW, suggesting increased mitochondrial biogenesis in the normoglycemic obese group. These mitochondria showed similar morphology to NW. On the contrary, in $\mathrm{OB} / \mathrm{GDM}(+)$, mtDNA was not significantly increased versus NW. Nevertheless, mitochondria showed morphological abnormalities, indicating impaired functionality. The metabolic response of the placenta to impairment in obese pregnancies can possibly vary depending on several parameters, resulting in opposite strains acting when insulin resistance of GDM occurs in the obese environment, characterized by inflammation and oxidative stress. Therefore, mitochondrial alterations represent a feature of obese pregnancies with changes in placental energetics that possibly can affect pregnancy outcomes.

\section{Introduction}

The placenta is a metabolically active organ with multiple functions connecting the mother and the fetus for a successful outcome of pregnancy [1].

Mitochondrial oxidative phosphorylation and substrate oxidation represent the main energy source for placental function [2]. Therefore, mitochondrial function or dysfunction plays an important role in metabolic health and cellular fate [3]. In the human and rodent placenta, both nutritional and hypoxic stresses can alter mitochondrial function [4-10], with changes in mitochondrial biogenesis, function, and morphology leading to placental dysfunction. Placental alterations can affect fetal metabolism and development possibly leading to higher risk of developing disease in the future adult [11].
In the last decade, obesity has become a global problem [12]. Maternal obesity (MO) is expanding exponentially worldwide to almost epidemic proportions, with an additional $5-10 \%$ of pregnant women with diabetes, representing a significant risk factor for adverse pregnancy outcomes [13-17] with both immediate and long-term consequences [11, 18-22]. However, molecular mechanisms underlying programming effects have been only partially identified. Impaired placental transfer and metabolism of energy substrates in maternal obesity and/or diabetes mellitus have been reported $[23,24]$. A lipotoxic placental environment is indeed recognized in maternal obesity, with an altered metabolome profile, increased inflammation and oxidative stress, and decreased regulators of angiogenesis [25-28]. This might alter mitochondrial function, resulting in excessive production of reactive oxygen species and oxidative 
stress, in a vicious cycle leading to placental dysfunction and impaired pregnancy outcomes.

In this study, we addressed the hypothesis that maternal pregestational body mass index (BMI) and glycemic levels can alter placental mitochondria, by measuring mitochondrial content and morphology in term placentas sampled at elective cesarean section.

\section{Materials and Methods}

Pregnant women were enrolled in the Unit of Obstetrics and Gynecology of the Luigi Sacco Hospital in Milan.

The study protocol was approved by the local Institutional Review Board (Luigi Sacco Hospital Ethical Committee), and all participants gave their informed consent.

2.1. Population. Only Caucasian women with singleton spontaneous pregnancy and delivering at term by elective cesarean section were included in this study. Cesarean sections before labor were performed for breech presentation, repeated caesarean section, or maternal request. Exclusion criteria were represented by maternal-fetal infections or autoimmune diseases, maternal smoking and drug-alcohol abuse, fetal malformations, chromosomal disorders, preeclampsia, and intrauterine growth restriction.

Forty-seven pregnant women were eligible for the study.

Thirty-seven presented normal glycemia values based on an oral glucose tolerance test (OGTT-75g) [29]. Among them, twenty-one were within normal weight $\left(\mathrm{NW} ; 18.5 \leq \mathrm{BMI}<25 \mathrm{~kg} / \mathrm{m}^{2}\right)$ and sixteen were obese (OB/ $\mathrm{GDM}(-) ; \mathrm{BMI} \geq 30 \mathrm{~kg} / \mathrm{m}^{2}$ ) according to their pregestational BMI [30].

Ten women were diagnosed with gestational diabetes mellitus (GDM) according to the OGTT at 24-28 weeks of gestation, and all of them were obese $(\mathrm{OB} / \mathrm{GDM}(+))$. Women with GDM underwent daily checks of glycemia.

All women were given nutritional and lifestyle advice and recommendations on weight gain during pregnancy following IOM guidelines, depending on pregestational BMI [30].

Obese patients had regular specific checkups in a dedicated antenatal clinic, where they received specific dietary indication to support the control of their gestational weight gain and their glycemia levels. No patient needed insulin therapy.

NW women had physiological pregnancies with a normal intrauterine growth and appropriate for gestational age birth weight according to reference ranges for the Italian population [31].

2.2. Data Collection. Maternal medical history, demographic, anthropometric, obstetric, and neonatal data were recorded at recruitment and after cesarean delivery.

Maternal hemoglobin was measured at 34-36 weeks. Maternal fasting glycemia was obtained from the first value of the OGTT performed between 24 and 28 weeks.

2.3. Sample Collection and Processing. Human placentas were collected immediately after elective cesarean section, in the absence of labor. Placentas were weighed after discarding membranes and cord from the disc, and biometric measurements were performed as previously described [18].

After removing the maternal decidua, placental biopsies $\left(\sim 1 \mathrm{~cm}^{3}\right)$ were sized from different cotyledons [32] midway between the cord insertion and placental border. Placental villi were then washed in PBS (Dulbecco's phosphatebuffered solution; Euroclone, Milano, Italy) and immediately frozen in liquid nitrogen to be stored at $-80^{\circ} \mathrm{C}$ until mtDNA analysis or alternatively were fixed with $2.5 \%$ glutaraldehyde for electron microscopy.

2.3.1. Placenta mtDNA Content. Frozen placental fragments (90 mg) were minced in a TRIzol reagent (Roche Diagnostics, Indianapolis, IN, USA) with a Potter homogenizer. Total DNA was isolated from this mixture with a chemical procedure, following the manufacturer's instructions. DNA concentrations were measured by a NanoDrop ND1000 spectrophotometer (NanoDrop Technologies; Wilmington, DE, USA).

Mitochondrial DNA (mtDNA) content was assessed by real-time PCR, normalizing levels of a mitochondrial gene (cytochrome- $\beta, \mathrm{CYB}$ ) to those of a single-copy nuclear gene (RNase-P) (2 $2^{-\Delta \mathrm{Cq}}$ method). Briefly, 30 nanograms of total DNA was analyzed in triplicate with TaqMan assays (CYB: Hs02596867_s1 and RNase-P: 4316844) by 7500 Fast RealTime PCR (Applied Biosystems, Thermo Fisher Scientific; Carlsbad, CA, USA); Cq values with standard deviation exceeding 0.25 were excluded.

2.3.2. Electron Microscopy. Cells were fixed with $2.5 \%$ glutaraldehyde in $100 \mathrm{mM}$ cacodylate buffer $\mathrm{pH} 7.4$ for 1 hour at room temperature. After several washes in cacodylate buffer, cells were postfixed with $1 \%$ osmium tetroxide and $1.5 \%$ potassium ferrocyanide in $100 \mathrm{mM}$ cacodylate buffer $\mathrm{pH} 7.4$ for 1 hour on ice. After a rinse in $\mathrm{dH}_{2} \mathrm{O}$, samples were en bloc stained in $0.5 \%$ uranyl acetate overnight and dehydrated in increasing concentrations of ethanol and finally embedded in Epon. Samples were cured at $60^{\circ} \mathrm{C}$ in an oven for $48 \mathrm{~h}$. Epon blocks were sectioned using a Leica EM UC7 ultramicrotome (Leica Microsystems, UK). Ultrathin sections $(70 \mathrm{~nm})$ were contrasted with $2 \%$ uranyl acetate and Sato's lead solutions and observed with a LEO 912AB Zeiss Transmission Electron Microscope (Carl Zeiss, Oberkochen, Germany). Digital micrographs were taken with a $2 \mathrm{k} \times 2 \mathrm{k}$ bottom-mounted slow-scan ProScan camera (ProScan, Lagerlechfeld, Germany) controlled by the EsivisionPro 3.2 software (Soft Imaging System, Münster, Germany).

2.4. Statistical Analyses. Data are presented as mean \pm standard error.

Maternal, fetal, and molecular data were compared among study groups by one-way analysis of variance (ANOVA), having preliminarily verified that no serious statistical violations occurred. Tukey HSD test was then run as a post hoc test.

A two-way between-group ANOVA was conducted to explore the impact of maternal BMI/GDM and fetal sex (independent variables), as individual or joint effect, on placental levels of mtDNA (dependent variable). 
TABLE 1: Maternal, fetal and placental characteristics in the study population.

\begin{tabular}{lccc}
\hline & $\mathrm{NW}, n=21$ & $\mathrm{OB} / \mathrm{GDM}(-), n=16$ & $\mathrm{OB} / \mathrm{GDM}(+), n=10$ \\
\hline Maternal data & $35.7 \pm 1.02$ & $32.7 \pm 1.26$ & $35.7 \pm 1.39$ \\
Age, years & $21.5 \pm 0.45$ & $34.9 \pm 1.17^{* * *}$ & $35.6 \pm 1.46^{* * *}$ \\
Pregestational BMI, kg/m ${ }^{2}$ & $81.4 \pm 1.72$ & $82.0 \pm 1.80$ & $90.4 \pm 4.13^{*}$ \\
Fasting glycemia, $\mathrm{mg} / \mathrm{dl}$ & $11.4 \pm 0.20$ & $10.7 \pm 0.34$ & $11.1 \pm 0.27$ \\
Hb, g/dl & $10.38 \pm 0.58$ & $7.94 \pm 1.06$ & $8.50 \pm 1.57$ \\
GWG, kg & $64.9 \pm 3.65$ & $95.5 \pm 9.18$ & $92.8 \pm 17.43$ \\
GWG to IOM advised limits, \% & & $39.1 \pm 0.07$ & $39.1 \pm 0.05$ \\
Fetal \& placental data at delivery & $39.2 \pm 0.12$ & $3339.1 \pm 87.8$ & $3394.0 \pm 126.0$ \\
Gestational age, weeks & $3329.6 \pm 63.1$ & $488.4 \pm 22.0$ & $559.0 \pm 20.5^{* *}$ \\
Fetal weight, g & $460.6 \pm 19.2$ & $7.00 \pm 0.30$ & $6.11 \pm 0.23^{*}$ \\
Placental weight, g & $7.54 \pm 0.41$ & $243.7 \pm 16.2$ & $259.3 \pm 17.7$ \\
Placental efficiency & $257.5 \pm 15.7$ & $2.12 \pm 0.15$ & $13.25 \pm 0.17$ \\
Placental area, cm & & $13.7 \pm 0.51$ \\
Placental thickness, cm & $1.84 \pm 0.12$ & $14.0 \pm 0.34$ & \\
Umbilical vein Hb, g/dl & & & \\
\hline
\end{tabular}

Data are presented as mean \pm standard error. Post hoc comparisons using the Tukey HSD test: ${ }^{*} p \leq 0.05,{ }^{* *} p \leq 0.01,{ }^{* * *} p \leq 0.001$ versus NW. NW: normal weight; OB/GDM(-): obese without a diagnosis of GDM; OB/GDM(+): obese with GDM; GDM: gestational diabetes mellitus; BMI: body mass index; maternal fasting glycemia: referred to the first value of the oral glucose tolerance test (OGTT); Hb: hemoglobin; GWG: gestational weight gain; IOM: Institute of Medicine; placental efficiency: fetal/placental weight ratio; placental area: (larger diameter $) \times($ smaller diameter $) \times(\pi / 4)$.

Chi-square analyses were performed to compare anemia frequencies among groups, using Yates continuity correction.

Correlations describing the strength and direction of the relationships between 2 parameters were assessed using the Pearson product-moment correlation.

All statistical tests were 2-sided, and $p$ values $<0.05$ were considered statistically significant. Statistical analysis was performed using SPSS (version 24.00, IBM Statistics; Armonk, NY, USA).

\section{Results}

3.1. Characteristics of the Study Population. Maternal, fetal and placental data are reported in Table 1.

According to inclusion criteria, pregestational BMI was significantly different among groups $(F(2,46)=78.52$, $p \leq 0.001$ ), being higher in the two obese groups compared to NW. Obese women gained on average less weight during pregnancy compared to normal weight, mostly remaining within IOM recommended limits for gestational weight gain during pregnancy of obese women [30]. As expected, maternal fasting glycemia was significantly different among groups $(F(2,46)=3.71, p=0.03)$, with $\mathrm{OB} / \mathrm{GDM}(+)$ showing higher levels compared to normoglycemic groups (Tukey HSD test, $p=0.03$ ). Hemoglobin levels were lower in the two OB groups compared to NW, though not significant. However, anemia $(\mathrm{Hb}<11.0 \mathrm{~g} / \mathrm{dl})$ frequency was higher in $\mathrm{OB}$, resulting more than two-fold higher in OB/GDM(-) (56\%) and in $\mathrm{OB} / \mathrm{GDM}(+)(50 \%)$ than in NW (25\%) subjects.

Maternal age, gestational age, and fetal weight did not differ among groups.

There was a statistically significant difference in placental weight $(F(2,46)=4.75, p=0.01)$. Post hoc comparisons using the Tukey HSD test indicated that the mean score for $\mathrm{OB} / \mathrm{GDM}(+)$ was significantly higher compared to the NW group $(p=0.01)$.

Placental efficiency (fetal/placental weight ratio) was significantly different among groups $(F(2,46)=3.29, p=0.04)$, with $\mathrm{OB} / \mathrm{GDM}(+)$ showing decreased placental efficiency compared to NW ( $p=0.03)$.

In our study population, placental efficiency was significantly and positively correlated with maternal $\mathrm{Hb}(p=$ $0.005, r=0.412$, Figure 1$)$ and with gestational age $(p=$ $0.001, r=0.457)$, while it was negatively correlated with placental thickness $(p=0.03, r=-0.336)$. Maternal $\mathrm{Hb}$ also positively correlated with gestational age $(p=0.004, r=$ $0.422)$ and negatively with placental weight $(p=0.03, r=$ -0.323) (data not shown).

Among the analyzed pregnancies, 23 carried male fetuses (15 of NW, 6 of OB/GDM(-), and 2 of OB/GDM(+) mothers) and 24 carried female fetuses (6 of NW, 10 of $\mathrm{OB} / \mathrm{GDM}(-)$, and 8 of $\mathrm{OB} / \mathrm{GDM}(+)$ mothers).

3.2. mtDNA Content in Placental Tissue. A one-way betweengroup analysis of variance was conducted to explore the impact of obesity and GDM on levels of mitochondrial DNA. There was a statistically significant difference among groups in mtDNA levels $(F(2,46)=3.03, p=0.49)$. Post hoc comparisons using the Tukey HSD test indicated that the mean score for the $\mathrm{OB} / \mathrm{GDM}(-)$ group was significantly higher compared to NW $(p=0.047)$, while $\mathrm{OB} / \mathrm{GDM}(+)$ was not (Figure 2).

Figure 3(a) shows the relation between mtDNA levels and pregestational BMI in the study population. There was a significant correlation in patients without a diagnosis of GDM, indicated by the regression line $(p=0.010, r=0.419)$, 


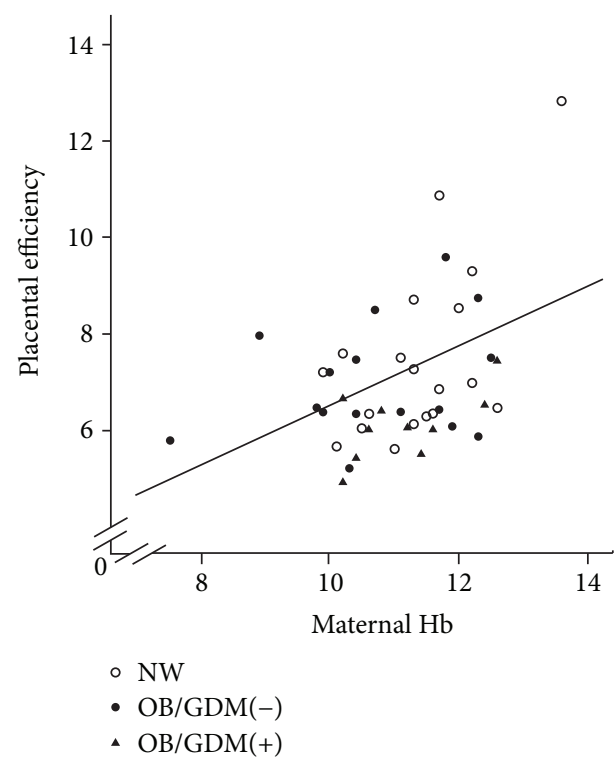

Figure 1: Significant correlation between placental efficiency and maternal hemoglobin $(p=0.005, r=0.412)$. NW: normal-weight women; $\mathrm{OB} / \mathrm{GDM}(-)$ : obese women without a diagnosis of GDM; $\mathrm{OB} / \mathrm{GDM}(+)$ : obese women with GDM.

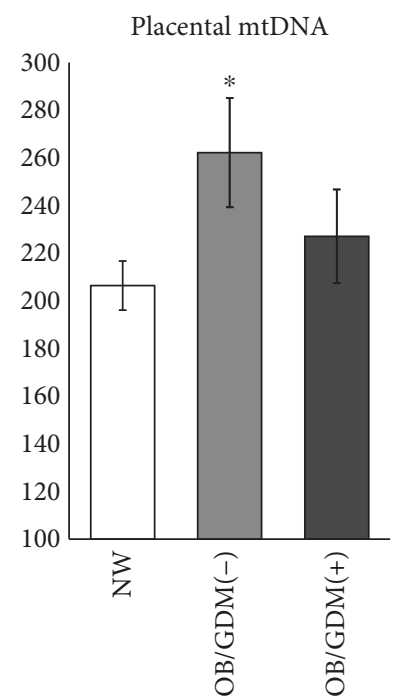

Figure 2: Placental mtDNA levels. ${ }^{*} p=0.047$ versus NW, Tukey HSD test. NW: normal-weight women; OB/GDM(-): obese women without a diagnosis of GDM; OB/GDM(+): obese women with GDM.

while no significant correlation was found in $\mathrm{OB}$ patients with GDM. mtDNA also negatively correlated with maternal $\mathrm{Hb}(p=0.011, r=-0.373)$ (Figure 3(b)) and umbilical vein $\mathrm{Hb}(p=0.019, r=-0.406)$ (Figure 3(c)).

There were no differences in mtDNA levels depending on fetal sex. The two-way between-group analysis of variance showed that the interaction effect between fetal sex and maternal pregestational BMI was not statistically significant $(F(2,46)=0.61, p=0.94)$ (data not shown).
3.3. Electron Microscopic Analysis of Syncytiotrophoblast. The mitochondrial profiles in the syncytiotrophoblast of both $\mathrm{NW}$ and $\mathrm{OB} / \mathrm{GDM}(-)$ placentas were round or elongated with a very dense matrix and similar structure of the cristae (Figures 4(a) and 4(b)). In contrast, syncytiotrophoblast mitochondria in $\mathrm{OB} / \mathrm{GDM}(+)$ placentas displayed morphological abnormalities, showing a matrix with very low density and vesicle-like or disrupted cristae, forming an irregular pattern (Figure 4(c)).

\section{Discussion}

Recently, maternal obesity has been associated with a lipotoxic placental environment, with increased placental lipids, inflammation, and oxidative stress, together with a less efficient fetal/placental ratio and altered metabolome profile $[18,23,25,27]$. The cellular stress characterizing this maternal environment may adversely affect placental development and function possibly altering fetal growth and development. Indeed, oxidative stress is one of the hallmark responses to intracellular lipid overload. High levels of free fatty acids impact the mitochondrial (mt) membrane structure, causing the release of reactive oxygen species (ROS) that can react with macromolecules and damage intracellular membranes and DNA [33]. These alterations can in turn affect mitochondrial structure and function, in a vicious cycle of mitochondrial abnormalities and ROS formation, possibly representing a key mechanism of placental dysfunction in a disease condition.

Several animal models of MO report mt dysfunctions in pancreatic islets, liver, or skeletal muscle of the offspring $[34,35]$. However, maternal obesity and diabetes are not always associated with obvious fetal distress, and the possible placental adaptation may explain it [24].

In this study, we addressed the hypothesis that placental mitochondria in pregnancy can be altered by elevated maternal BMI and/or by metabolic alterations occurring in gestational diabetes mellitus.

We studied placentas at term only delivered by elective cesarean section, in order to avoid possible alterations of mitochondrial content or function due to labor [36]. Obese patients were followed during pregnancy with a specific counseling including nutritional and lifestyle advices. This resulted in lower gestational weight gain compared to normal weight patients, as recommended by IOM guidelines [30]. Our study population was also carefully selected by excluding women carrying further conditions possibly affecting mitochondrial characteristics, such as maternal smoking or drug-alcohol abuse and maternal or fetal pathologies. Obese women with GDM were included, in order to evaluate the additional effect of increased glycemia to placental mitochondrial features.

4.1. mtDNA in Obese Pregnancies without GDM. mtDNA levels are largely recognized as a measure of the mitochondrial content $[4,37,38]$. We found higher levels of mtDNA, accounting for the increased mitochondrial content in placental cells of women with an obese pregestational BMI without a diagnosis of GDM (Figure 2). The morphology of 


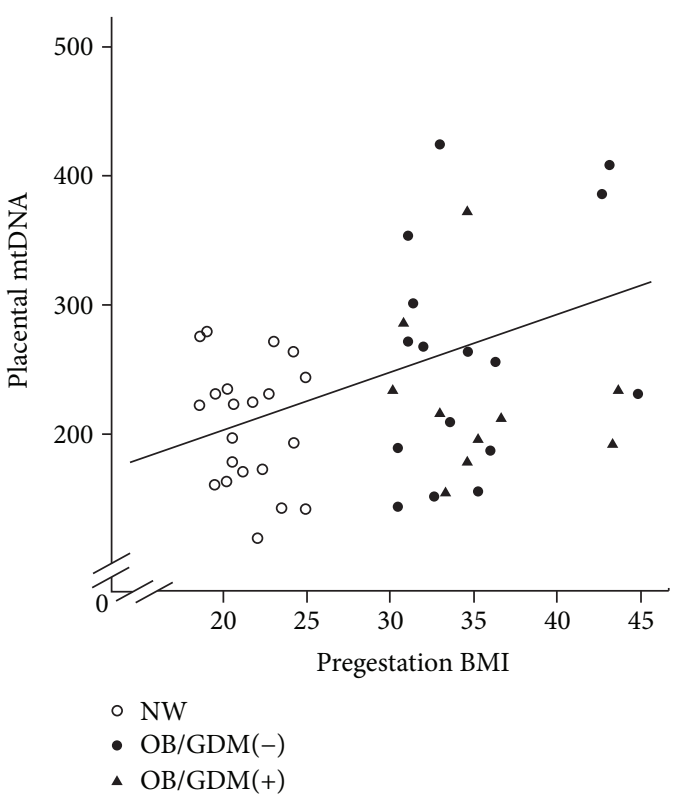

(a)

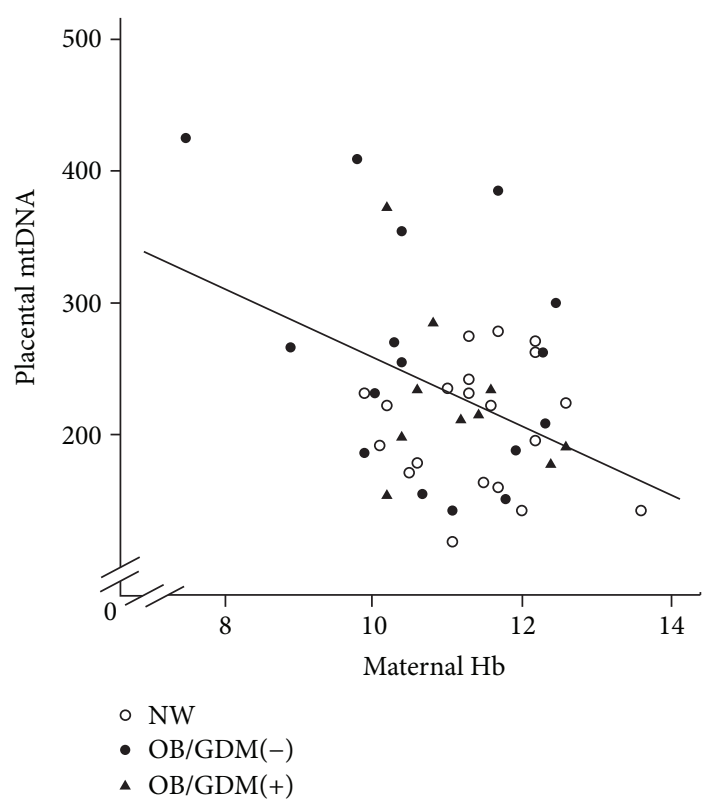

(b)

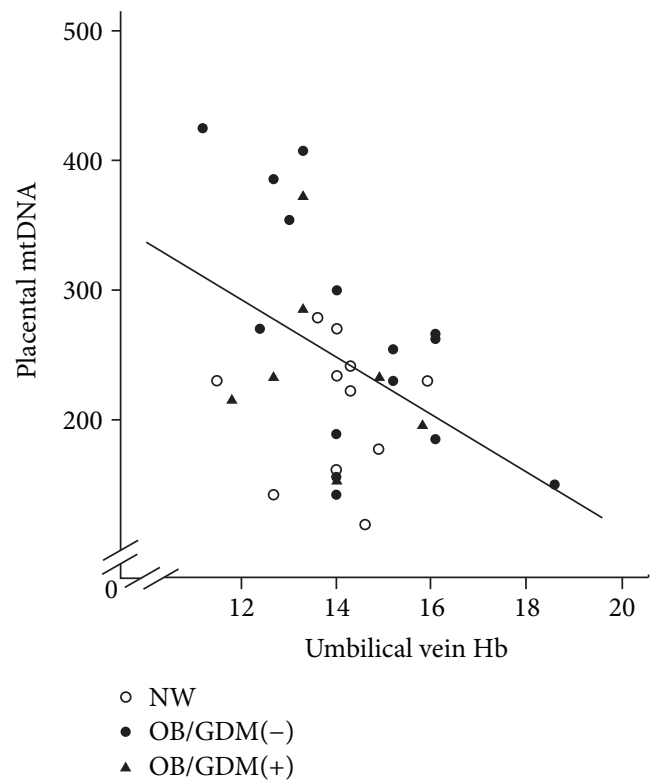

(c)

FIgURE 3: (a) Correlation between placental mtDNA and maternal pregestational BMI. The correlation is significant in patients without a diagnosis of GDM, indicated by the regression line $(p=0.010, r=0.419)$. (b). Correlation between placental mtDNA and maternal hemoglobin $(p=0.011, r=-0.373)$. (c). Correlation between placental mtDNA and umbilical vein hemoglobin $(p=0.019, r=-0.406)$. NW: normal-weight women; OB/GDM(-): obese women without a diagnosis of GDM; OB/GDM(+): obese women with GDM.

mitochondria in the syncytiotrophoblast of the OB/GDM(-) group was overall similar to the NW group, suggesting no alterations in mitochondrial function (Figures 4(a) and 4(b)). A compensatory increase in mitochondrial biogenesis can be explained by the endocrine stimuli due to high intracellular fatty acid levels and oxidative stress occurring in the lipotoxic environment of obese placentas $[9,34]$. Indeed, altered levels of mtDNA as well as the impairment of nutrient transport systems have been reported in previous studies in the placental tissue of different pregnancy pathologies characterized by elevated oxidative stress and inflammation levels, such as intrauterine growth restriction and preeclampsia $[4,5,39-43]$. The positive correlation between placental mtDNA and maternal BMI that was observed in this study supports this hypothesis (Figure 3(a)).

Differently from our study, decreased mtDNA copy number has been previously reported in placentas of obese compared to not-obese women $[9,44]$. However, different gene assays and different population criteria were employed in these studies. One of the strengths of the present study is 


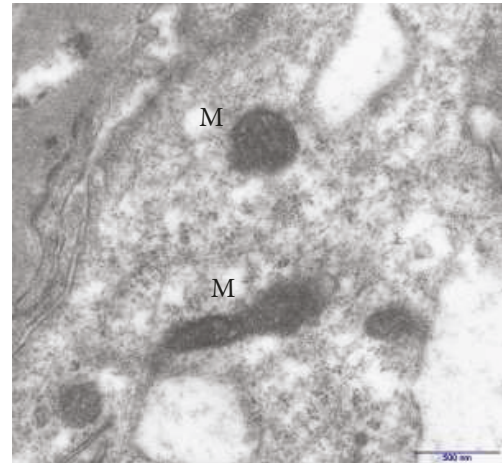

(a)

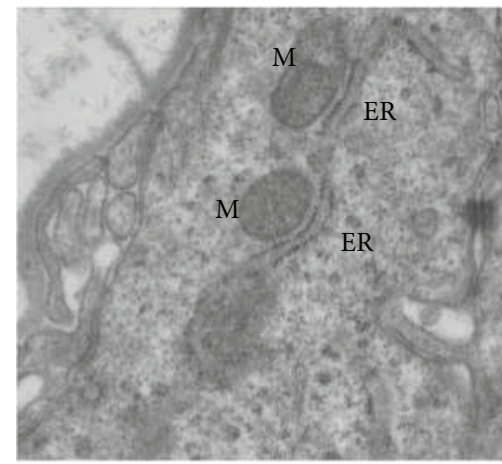

(b)

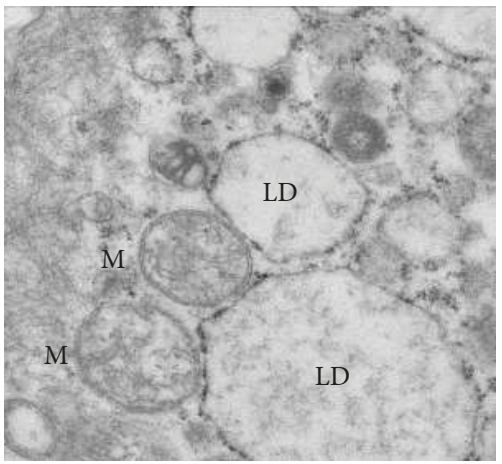

(c)

FIGURE 4: Electron microscopy of term placenta villi showing representative sections of syncytiotrophobast cells from NW (a), OB/GDM(-) (b), and $\mathrm{OB} / \mathrm{GDM}(+)$ (c) term placentas. M: mitochondria; ER: endoplasmic reticulum; LD: lipid droplet. NW: normal-weight women; OB/ $\operatorname{GDM}(-)$ : obese women without a diagnosis of GDM; OB/GDM(+): obese women with GDM.

the careful selection of a very well characterized population of uncomplicated pregnancies. We excluded any maternal or fetal infection or autoimmune disease, maternal smoking or drug-alcohol abuse, fetal malformations, chromosomal disorders, preeclampsia, and intrauterine growth restriction, all of which can affect mitochondrial biogenesis and functionality. Moreover, only Caucasian women were selected, as different mitochondrial DNA haplogroups have been identified and have been suggested to possibly contribute to the genetic component of complex disorders [45-47]. Finally, all women included in this study were counseled with nutritional and lifestyle advice and recommendations on weight gain during pregnancy, and obese patients had regular specific checkups in a dedicated antenatal clinic with specific dietary indication.

Interestingly, in a recent review on mitochondrial features in gestational disorders, Holland and colleagues reported that mitochondrial content has been found to be increased or decreased in the same pregnancy pathology by different studies [48]. These apparent differences within the same pathologies can be explained by the different severity or timing of the insult and the resulting capacity of the tissue to respond. Indeed, although in several diseases, mitochondrial biogenesis is thought to occur as a compensatory mechanism to the cell distress $[4,49-52]$, on the other hand, the increase of mitochondrial ROS production could damage the mitochondrial DNA and membranes, thus inhibiting the adaptive mitochondrial increase.

Therefore, the different inclusion/exclusion criteria of the studied population, together with different clinical protocols in nutritional and lifestyle advice, can lead to different results regarding the mitochondrial responses. This is also suggested in the recent study reporting increased placental mitochondrial content in early-onset but not late-onset preeclampsia [41].

4.2. mtDNA in Obese Pregnancies with GDM. When analyzing placentas of obese women with GDM, we did not find a significant increase in mtDNA levels (Figure 2). However, placentas of $\mathrm{OB} / \mathrm{GDM}(+)$ women showed dysfunctional syncytiotrophoblast mitochondria, with morphological abnormalities. In particular, electron microscopy revealed a loss of matrix density and disorganization of inner membrane cristae (Figure 4(c)). Interestingly, also animal models of diabetes showed a reduced number of mitochondria, with abnormal morphology, associated to mitochondrial dysfunction [53]. Notably, large lipid droplets that stock lipids as energy-rich storage compounds were observed. In this context, lipid droplets may support feeble mitochondrial function by supplying fatty acids for mitochondrial $\beta$ oxidation and protect mitochondria from lipotoxicity [54].

Our results therefore suggest that placental mitochondria of obese women with GDM do not show significant alterations in biogenesis but present altered morphology, indicating an impairment of their function.

The OB/GDM(+) group also presented decreased placental efficiency compared to NW. Indeed, GDM has been associated with impaired placental development showing villous immaturity or alterations in villous branching, as well as impaired placental angiogenesis, villous vasculature, and uteroplacental perfusion [55-57]. Oxidative stress markers have also been reported in GDM placentas [58], possibly affecting the physiology of the placental vasculature and mitochondrial morphology.

Insulin resistance and altered metabolic profiles characterize the diabetic condition. Insulin resistance has been correlated in several tissues with a decrease in mitochondrial function and mitochondrial DNA copy number, a reduction in mitochondrial fusion and increase in their fission and with alterations of mitochondrial size and density. The possible role of epigenetic regulation is emerging for these alterations [59-64]. Indeed, some authors recently hypothesized that insulin resistance acts on the expression of proteins involved in the methylation machinery of both nuclear and mitochondrial DNA, affecting the expression of genes involved in mtDNA replication, thus leading to decreased mitochondrial biogenesis $[62,65,66]$. However, other studies report either no impairment or a compensatory increase of mitochondrial function and oxidative capacity in conditions of insulin resistance $[67,68]$. Hence, the relationship between 
mitochondria and insulin action is highly complex and there is still much to learn in this area [3].

Similarly to our results, decreased levels of placental mtDNA have been recently reported in diabetic pregnancies [69], together with lower mitochondrial respiratory chain enzyme activities [9]. In our population, different levels of placental mtDNA in obese with or without GDM may be the result of opposite strains. Maternal diabetes has been associated with a decrease of placental mitochondrial levels $[9,69]$, while the obese environment associated with inflammation and oxidative stress tends to promote a compensatory mitochondrial biogenesis. In the recent study, we reported comparable results in the maternal blood of obese women with or without GDM [52]. Levels of mtDNA in maternal blood may indeed result by the release of placental cell debris in the maternal circulation [70].

In addition, complex changes in the metabolic profile have been shown in obese pregnant women with or without GDM $[25,71]$. White et al. recently showed that in addition to the dysregulation of glucose metabolism, GDM obese women compared with non-GDM obese women exhibited exaggerated dyslipidemic profiles prior to the GDM diagnosis, at week 17, when placentation still occurs. This possibly reflects enhanced insulin resistance in peripheral tissues of GDM women and a consequent reduced suppression of lipolysis, affecting lipid metabolism pathways. Increased insulin resistance and higher levels of lipids and lipoproteins have also been shown at mid and late pregnancy in women with GDM compared to normal glucose tolerant patients [72].

Moreover, GDM-obese women showed metabolic patterns consistent with perturbed energy pathways $[25,71]$. Particularly, obese women with GDM showed increased levels of acetoacetate (likely secondary to unregulated fatty acid oxidation in mitochondria) and citrate (an early intermediate of the tricarboxylic acid cycle, occurring in mitochondria). These evidences suggest a specific metabolic milieu of GDM compared to non-GDM obese pregnant women, which can differently affect the mitochondrial function in placenta.

4.3. Relation between mtDNA and Maternal/Fetal Hemoglobin. Noteworthy, in our study population, we found a significant negative correlation between placental mtDNA and hemoglobin levels in the maternal and fetal blood (Figures 3(b) and 3(c)). Maternal $\mathrm{Hb}$ also significantly correlated with placental efficiency.

Maternal systemic hemoglobin may account for maternal nutritional status. Obese women in this study did not present significantly lower levels of maternal $\mathrm{Hb}$, possibly due to the specific and regular nutritional counseling given to them during pregnancy. However, in our population anemia frequency $(\mathrm{Hb}<11.0 \mathrm{~g} / \mathrm{dl})$ was higher in both $\mathrm{OB}$ subgroups compared to NW. Indeed, obesity induces a chronic, lowgrade inflammation with overexpression of C-reactive protein and hepcidin $[26,52]$ that is negatively correlated with both maternal and cord blood iron status [73]. On the other hand, low maternal $\mathrm{Hb}$ may be an index of altered vascular oxygenation that can induce mitochondrial biogenesis, according to the negative correlation we found in our study population.

4.4. Limitations. A sexual dimorphism has been reported in several placental responses to adverse environments $[13,18,74]$. Nevertheless, in our study population, we did not find any interaction effect between the fetal sex and maternal pregestational BMI, suggesting that the BMI influence on mtDNA levels and morphology does not depend on fetal sex. However, a limited number of cases within each subgroup might lead to these results. Therefore, further investigations are needed to explore the possible effect of fetal sex on the mitochondrial content and function in placentas of obese pregnancies with or without GDM.

Although our results on placental mitochondria of $\mathrm{OB} / \mathrm{GDM}(+)$ pregnancies comply with previous findings, showing lower mitochondrial copy number compared to NW placentas $[9,69]$, inclusion criteria of the populations analyzed in these studies were different to ours, also including type 1 and type 2 diabetes mellitus, placentas from vaginal deliveries, and different ethnic groups, thus keeping open the need of further studies investigating the consequences of insulin action on placental mitochondria in GDM pregnancies.

Moreover, whether alterations in mtDNA content of OB placental whole tissue are due to alterations in one or more placental cell types still remains to be investigated.

\section{Conclusions}

The placenta integrates nutritional and endocrine signals and arranges its metabolic phenotype to support pregnancy. The metabolic response of the placenta to impairment depends on the nature, severity, and duration of the environmental adversity [1], and in obese pregnancies, these can possibly vary depending on several parameters like maternal glycemia or the maternal nutritional status and lifestyle. However, mitochondrial alterations are a clear feature of obese pregnancies with changes in placental energetics and consumption of oxidative substrates that possibly can affect fetal delivery of nutrients and $\mathrm{O}_{2}$ with short- and long-term consequences on the newborn.

Understanding the differences in placental metabolic adaptation to obesity and insulin resistance might open new perspectives for therapeutic future developments [24].

\section{Data Availability}

Readers may access the data underlying the findings of the study by writing to the corresponding author.

\section{Conflicts of Interest}

The authors declare that there is no conflict of interest regarding the publication of this article.

\section{Acknowledgments}

This work was financially supported by a grant from MIUR: PRIN 2010-2011 prot. 20102chst5_005 "Parto pre-termine: 
markers molecolari, biochimici e biofisici dell'unità fetoplacentare" (to Irene Cetin). The authors are thankful to Fondazione Ricerca Donna e Feto onlus and to ASM (Associazione Studio Malformazioni) for an unconditioned grant to the Laboratory of Maternal-Fetal Translational Research "Giorgio Pardi."

\section{References}

[1] O. R. Vaughan and A. L. Fowden, "Placental metabolism: substrate requirements and the response to stress," Reproduction in Domestic Animals, vol. 51, pp. 25-35, 2016.

[2] B. E. Bax and D. L. Bloxam, "Energy metabolism and glycolysis in human placental trophoblast cells during differentiation," Biochimica et Biophysica Acta (BBA) - Bioenergetics, vol. 1319, no. 2-3, pp. 283-292, 1997.

[3] M. K. Montgomery and N. Turner, "Mitochondrial dysfunction and insulin resistance: an update," Endocrine Connections, vol. 4, no. 1, pp. R1-R15, 2015.

[4] C. Mandò, C. De Palma, T. Stampalija et al., "Placental mitochondrial content and function in intrauterine growth restriction and preeclampsia," American Journal of PhysiologyEndocrinology and Metabolism, vol. 306, no. 4, pp. E404E413, 2014.

[5] C. Mandò, P. Razini, C. Novielli et al., "Impaired angiogenic potential of human placental mesenchymal stromal cells in intrauterine growth restriction," Stem Cells Translational Medicine, vol. 5, no. 4, pp. 451-463, 2016.

[6] F. Colleoni, N. Padmanabhan, H. W. Yung et al., "Suppression of mitochondrial electron transport chain function in the hypoxic human placenta: a role for miRNA-210 and protein synthesis inhibition," PLoS One, vol. 8, no. 1, article e55194, 2013.

[7] M. R. Chiaratti, S. Malik, A. Diot et al., "Is placental mitochondrial function a regulator that matches fetal and placental growth to maternal nutrient intake in the mouse?," PLoS One, vol. 10, no. 7, article e0130631, 2015.

[8] S. Mayeur, S. Lancel, N. Theys et al., "Maternal calorie restriction modulates placental mitochondrial biogenesis and bioenergetic efficiency: putative involvement in fetoplacental growth defects in rats," American Journal of PhysiologyEndocrinology and Metabolism, vol. 304, no. 1, pp. E14E22, 2013.

[9] R. Hastie and M. Lappas, "The effect of pre-existing maternal obesity and diabetes on placental mitochondrial content and electron transport chain activity," Placenta, vol. 35, no. 9, pp. 673-683, 2014.

[10] H. J. Rebelato, M. A. M. Esquisatto, C. Moraes, M. E. C. Amaral, and R. Catisti, "Gestational protein restriction induces alterations in placental morphology and mitochondrial function in rats during late pregnancy," Journal of Molecular Histology, vol. 44, no. 6, pp. 629-637, 2013.

[11] D. J. P. Barker, K. L. Thornburg, C. Osmond, E. Kajantie, and J. G. Eriksson, "Beyond birthweight: the maternal and placental origins of chronic disease," Journal of Developmental Origins of Health and Disease, vol. 1, no. 6, pp. 360-364, 2010.

[12] NCD Risk Factor Collaboration (NCD-RisC), "Worldwide trends in diabetes since 1980: a pooled analysis of 751 population-based studies with 4.4 million participants," The Lancet, vol. 387, no. 10027, pp. 1513-1530, 2016.
[13] L. Myatt and A. Maloyan, "Obesity and placental function," Seminars in Reproductive Medicine, vol. 34, no. 1, pp. 4249, 2016.

[14] H. D. McIntyre, K. S. Gibbons, V. J. Flenady, and L. K. Callaway, "Overweight and obesity in Australian mothers: epidemic or endemic?," The Medical Journal of Australia, vol. 196, no. 3, pp. 184-188, 2012.

[15] A. M. Stuebe, M. B. Landon, Y. Lai et al., "Maternal BMI, glucose tolerance, and adverse pregnancy outcomes," American Journal of Obstetrics and Gynecology, vol. 207, no. 1, pp. 62.e1-62.e7, 2012.

[16] L. Guariguata, U. Linnenkamp, J. Beagley, D. R. Whiting, and N. H. Cho, "Global estimates of the prevalence of hyperglycaemia in pregnancy," Diabetes Research and Clinical Practice, vol. 103, no. 2, pp. 176-185, 2014.

[17] A. Yessoufou and K. Moutairou, "Maternal diabetes in pregnancy: early and long-term outcomes on the offspring and the concept of "metabolic memory"," Experimental Diabetes Research, vol. 2011, Article ID 218598, 12 pages, 2011.

[18] C. Mandò, S. Calabrese, M. I. Mazzocco et al., "Sex specific adaptations in placental biometry of overweight and obese women," Placenta, vol. 38, pp. 1-7, 2016.

[19] E. A. Nohr, M. Vaeth, J. L. Baker, T. I. A. Sørensen, J. Olsen, and K. M. Rasmussen, "Combined associations of prepregnancy body mass index and gestational weight gain with the outcome of pregnancy," The American Journal of Clinical Nutrition, vol. 87, no. 6, pp. 1750-1759, 2008.

[20] P. Catalano and S. H. deMouzon, "Maternal obesity and metabolic risk to the offspring: why lifestyle interventions may have not achieved the desired outcomes," International Journal of Obesity, vol. 39, no. 4, pp. 642-649, 2015.

[21] P. M. Catalano, "Obesity and pregnancy - the propagation of a viscous cycle?," The Journal of Clinical Endocrinology and Metabolism, vol. 88, no. 8, pp. 3505-3506, 2003.

[22] T. Forsen, J. G. Eriksson, J. Tuomilehto, K. Teramo, C. Osmond, and D. J. P. Barker, "Mother's weight in pregnancy and coronary heart disease in a cohort of Finnish men: follow up study," BMJ, vol. 315 , no. 7112 , pp. $837-$ 840, 1997.

[23] I. Cetin, F. Parisi, C. Berti, C. Mando, and G. Desoye, "Placental fatty acid transport in maternal obesity," Journal of Developmental Origins of Health and Disease, vol. 3, no. 6, pp. 409-414, 2012.

[24] L. A. Gallo, H. L. Barrett, and M. Dekker Nitert, "Review: placental transport and metabolism of energy substrates in maternal obesity and diabetes," Placenta, vol. 54, pp. 5967, 2017.

[25] C. Fattuoni, C. Mandò, F. Palmas et al., "Preliminary metabolomics analysis of placenta in maternal obesity," Placenta, vol. 61, pp. 89-95, 2018.

[26] M. Zambon, C. Mandò, A. Lissoni et al., "Inflammatory and oxidative responses in pregnancies with obesity and periodontal disease," Reproductive Sciences, vol. 1, 2018.

[27] J. Saben, F. Lindsey, Y. Zhong et al., "Maternal obesity is associated with a lipotoxic placental environment," Placenta, vol. 35, no. 3, pp. 171-177, 2014.

[28] K. R. Howell and T. L. Powell, "Effects of maternal obesity on placental function and fetal development," Reproduction, vol. 153, no. 3, pp. R97-R108, 2016. 
[29] M. Hod, A. Kapur, D. A. Sacks et al., "The International Federation of Gynecology and Obstetrics (FIGO) initiative on gestational diabetes mellitus: a pragmatic guide for diagnosis, management, and care," International Journal of Gynaecology and Obstetrics, vol. 131, Supplement 3, pp. S173-S211, 2015.

[30] Institute of Medicine (US) and National Research Council (US). Committee to Reexamine IOM Pregnancy Weight Guidelines, Weight Gain during Pregnancy: Reexamining the Guidelines, K. M. Rasmussen and A. L. Yaktine, Eds., National Academies Press, Washington, DC, 2009.

[31] E. Bertino, E. Spada, L. Occhi et al., "Neonatal anthropometric charts: the Italian neonatal study compared with other European studies," Journal of Pediatric Gastroenterology and Nutrition, vol. 51, no. 3, pp. 353-361, 2010.

[32] T. M. Mayhew, "Taking tissue samples from the placenta: an illustration of principles and strategies," Placenta, vol. 29, no. 1, pp. 1-14, 2008.

[33] A. Agarwal, S. Gupta, L. Sekhon, and R. Shah, "Redox considerations in female reproductive function and assisted reproduction: from molecular mechanisms to health implications," Antioxidants \& Redox Signaling, vol. 10, no. 8, pp. 1375-1404, 2008.

[34] S. J. Borengasser, F. Lau, P. Kang et al., "Maternal obesity during gestation impairs fatty acid oxidation and mitochondrial SIRT3 expression in rat offspring at weaning," PLoS One, vol. 6, no. 8, article e24068, 2011.

[35] B. Reusens, N. Theys, and C. Remacle, "Alteration of mitochondrial function in adult rat offspring of malnourished dams," World Journal of Diabetes, vol. 2, no. 9, pp. 149157, 2011.

[36] O. J. Holland, A. J. R. Hickey, A. Alvsaker et al., "Changes in mitochondrial respiration in the human placenta over gestation," Placenta, vol. 57, pp. 102-112, 2017.

[37] D. P. Kelly and R. C. Scarpulla, "Transcriptional regulatory circuits controlling mitochondrial biogenesis and function," Genes \& Development, vol. 18, no. 4, pp. 357-368, 2004.

[38] M. A. Williams, S. E. Sanchez, C. V. Ananth, K. Hevner, C. Qiu, and D. A. Enquobahrie, "Maternal blood mitochondrial DNA copy number and placental abruption risk: results from a preliminary study," International Journal of Molecular Epidemiology and Genetics, vol. 4, no. 2, pp. 120-127, 2013.

[39] Z. Shi, W. Long, C. Zhao, X. Guo, R. Shen, and H. Ding, "Comparative proteomics analysis suggests that placental mitochondria are involved in the development of preeclampsia," PLoS One, vol. 8, no. 5, article e64351, 2013.

[40] Y. Wang and S. W. Walsh, "Placental mitochondria as a source of oxidative stress in pre-eclampsia," Placenta, vol. 19, no. 8, pp. 581-586, 1998.

[41] P. A. Vishnyakova, M. A. Volodina, N. V. Tarasova et al., "Mitochondrial role in adaptive response to stress conditions in preeclampsia," Scientific Reports, vol. 6, no. 1, article 32410, 2016.

[42] C. Mandò, S. Tabano, P. Pileri et al., "SNAT2 expression and regulation in human growth-restricted placentas," Pediatric Research, vol. 74, no. 2, pp. 104-110, 2013.

[43] C. Mandò, S. Tabano, P. Colapietro et al., "Transferrin receptor gene and protein expression and localization in human IUGR and normal term placentas," Placenta, vol. 32, no. 1, pp. 44-50, 2011.

[44] J. Mele, S. Muralimanoharan, A. Maloyan, and L. Myatt, "Impaired mitochondrial function in human placenta with increased maternal adiposity," American Journal of Physiology-Endocrinology and Metabolism, vol. 307, no. 5, pp. E419-E425, 2014.

[45] G. J. Tranah, T. M. Manini, K. K. Lohman et al., "Mitochondrial DNA variation in human metabolic rate and energy expenditure," Mitochondrion, vol. 11, no. 6, pp. 855-861, 2011.

[46] M. C. Kenney, M. Chwa, S. R. Atilano et al., "Molecular and bioenergetic differences between cells with African versus European inherited mitochondrial DNA haplogroups: implications for population susceptibility to diseases," Biochimica et Biophysica Acta (BBA) - Molecular Basis of Disease, vol. 1842, no. 2, pp. 208-219, 2014.

[47] A. Gómez-Durán, D. Pacheu-Grau, E. López-Gallardo et al., "Unmasking the causes of multifactorial disorders: OXPHOS differences between mitochondrial haplogroups," Human Molecular Genetics, vol. 19, no. 17, pp. 3343-3353, 2010.

[48] O. Holland, M. Dekker Nitert, L. A. Gallo, M. Vejzovic, J. J. Fisher, and A. V. Perkins, "Review: placental mitochondrial function and structure in gestational disorders," Placenta, vol. 54, pp. 2-9, 2017.

[49] H.-C. Lee, P.-H. Yin, C.-Y. Lu, C.-W. Chi, and Y.-H. Wei, "Increase of mitochondria and mitochondrial DNA in response to oxidative stress in human cells," The Biochemical Journal, vol. 348, no. 2, pp. 425-432, 2000.

[50] K. S. Sitarz, P. Yu-Wai-Man, A. Pyle et al., "MFN2 mutations cause compensatory mitochondrial DNA proliferation," Brain, vol. 135, no. 8, article e219, 2012.

[51] C. Novielli, C. Mandò, S. Tabano et al., "Mitochondrial DNA content and methylation in fetal cord blood of pregnancies with placental insufficiency," Placenta, vol. 55, pp. 63-70, 2017.

[52] G. M. Anelli, M. Cardellicchio, C. Novielli et al., "Mitochondrial content and hepcidin are increased in obese pregnant mothers," The Journal of Maternal-Fetal \& Neonatal Medicine, vol. 31, no. 18, pp. 2388-2395, 2017.

[53] Z. Ma, T. Wirström, L. A. H. Borg et al., "Diabetes reduces $\beta$-cell mitochondria and induces distinct morphological abnormalities, which are reproducible by high glucose in vitro with attendant dysfunction," Islets, vol. 4, no. 3, pp. 233-242, 2014.

[54] T. B. Nguyen, S. M. Louie, J. R. Daniele et al., "DGAT1-dependent lipid droplet biogenesis protects mitochondrial function during starvation-induced autophagy," Developmental Cell, vol. 42, no. 1, pp. 9-21.e5, 2017.

[55] J. Loegl, E. Nussbaumer, S. Cvitic, B. Huppertz, G. Desoye, and U. Hiden, "GDM alters paracrine regulation of feto-placental angiogenesis via the trophoblast," Laboratory Investigation, vol. 97, no. 4, pp. 409-418, 2017.

[56] M. Gauster, G. Desoye, M. Tötsch, and U. Hiden, "The placenta and gestational diabetes mellitus," Current Diabetes Reports, vol. 12, no. 1, pp. 16-23, 2012.

[57] R. Madazli, A. Tuten, Z. Calay, H. Uzun, S. Uludag, and V. Ocak, "The incidence of placental abnormalities, maternal and cord plasma malondialdehyde and vascular endothelial growth factor levels in women with gestational diabetes mellitus and nondiabetic controls," Gynecologic and Obstetric Investigation, vol. 65, no. 4, pp. 227-232, 2008.

[58] M. T. Coughlan, P. P. Vervaart, M. Permezel, H. M. Georgiou, and G. E. Rice, "Altered placental oxidative stress status in gestational diabetes mellitus," Placenta, vol. 25, no. 1, pp. 7884, 2004. 
[59] B. B. Lowell and G. I. Shulman, "Mitochondrial dysfunction and type 2 diabetes," Science, vol. 307, no. 5708, pp. 384387, 2005.

[60] T. F. Gianotti, S. Sookoian, G. Dieuzeide et al., "A decreased mitochondrial DNA content is related to insulin resistance in adolescents," Obesity, vol. 16, no. 7, pp. 1591-1595, 2008.

[61] S. Sookoian, M. S. Rosselli, C. Gemma et al., "Epigenetic regulation of insulin resistance in nonalcoholic fatty liver disease: impact of liver methylation of the peroxisome proliferator-activated receptor $\gamma$ coactivator $1 \alpha$ promoter," Hepatology, vol. 52, no. 6, pp. 1992-2000, 2010.

[62] L. D. Zheng, L. E. Linarelli, L. Liu et al., "Insulin resistance is associated with epigenetic and genetic regulation of mitochondrial DNA in obese humans," Clinical Epigenetics, vol. 7, no. 1, p. 60, 2015.

[63] N. Diaz-Morales, S. Rovira-Llopis, C. Bañuls et al., "Are mitochondrial fusion and fission impaired in leukocytes of type 2 diabetic patients?" Antioxidants \& Redox Signaling, vol. 25, no. 2, pp. 108-115, 2016.

[64] K. Morino, K. F. Petersen, S. Dufour et al., "Reduced mitochondrial density and increased IRS-1 serine phosphorylation in muscle of insulin-resistant offspring of type 2 diabetic parents," Journal of Clinical Investigation, vol. 115, no. 12, pp. 3587-3593, 2005.

[65] L. D. Zheng, L. E. Linarelli, J. Brooke et al., "Mitochondrial epigenetic changes link to increased diabetes risk and earlystage prediabetes indicator," Oxidative Medicine and Cellular Longevity, vol. 2016, Article ID 5290638, 10 pages, 2016.

[66] S. Tewari, Q. Zhong, J. M. Santos, and R. A. Kowluru, "Mitochondria DNA replication and DNA methylation in the metabolic memory associated with continued progression of diabetic retinopathy," Investigative Ophthalmology \& Visual Science, vol. 53, no. 8, pp. 4881-4888, 2012.

[67] N. Turner, C. R. Bruce, S. M. Beale et al., "Excess lipid availability increases mitochondrial fatty acid oxidative capacity in muscle: evidence against a role for reduced fatty acid oxidation in lipid-induced insulin resistance in rodents," Diabetes, vol. 56, no. 8, pp. 2085-2092, 2007.

[68] J. de Wilde, R. Mohren, S. van den Berg et al., "Short-term high fat-feeding results in morphological and metabolic adaptations in the skeletal muscle of C57BL/6J mice," Physiological Genomics, vol. 32, no. 3, pp. 360-369, 2008.

[69] S. Jiang, A. M. Teague, J. B. Tryggestad, C. E. Aston, T. Lyons, and S. D. Chernausek, "Effects of maternal diabetes and fetal sex on human placenta mitochondrial biogenesis," Placenta, vol. 57, pp. 26-32, 2017.

[70] A. E. P. Heazell, S. J. Moll, C. J. P. Jones, P. N. Baker, and I. P. Crocker, "Formation of syncytial knots is increased by hyperoxia, hypoxia and reactive oxygen species," Placenta, vol. 28, pp. S33-S40, 2007.

[71] S. L. White, D. Pasupathy, N. Sattar et al., "Metabolic profiling of gestational diabetes in obese women during pregnancy," Diabetologia, vol. 60, no. 10, pp. 1903-1912, 2017.

[72] Y. Zhang, H. H. Zhang, J. H. Lu et al., "Changes in serum adipocyte fatty acid-binding protein in women with gestational diabetes mellitus and normal pregnant women during mid- and late pregnancy," Journal of Diabetes Investigation, vol. 7, no. 5, pp. 797-804, 2016.
[73] M. C. Dao, S. Sen, C. Iyer, D. Klebenov, and S. N. Meydani, "Obesity during pregnancy and fetal iron status: is Hepcidin the link?," Journal of Perinatology, vol. 33, no. 3, pp. 177181, 2013.

[74] V. L. Clifton, "Review: sex and the human placenta: mediating differential strategies of fetal growth and survival," Placenta, vol. 31, pp. S33-S39, 2010. 


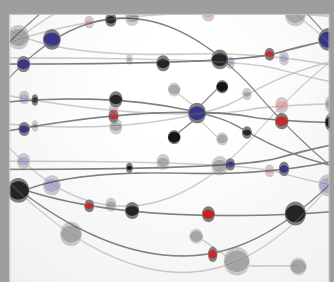

The Scientific World Journal
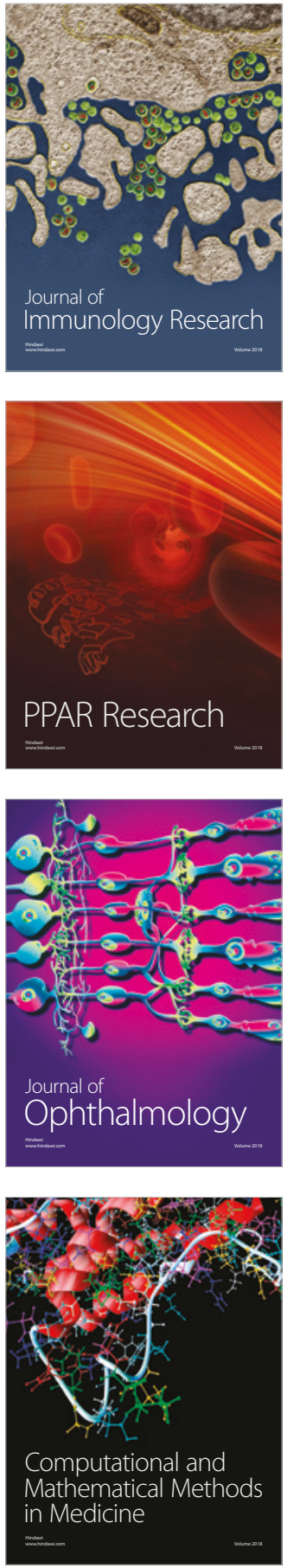

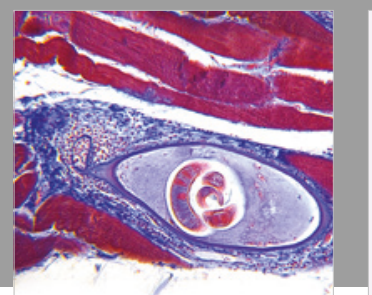

Gastroenterology Research and Practice

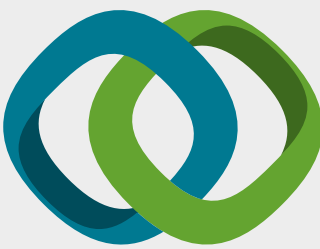

\section{Hindawi}

Submit your manuscripts at

www.hindawi.com
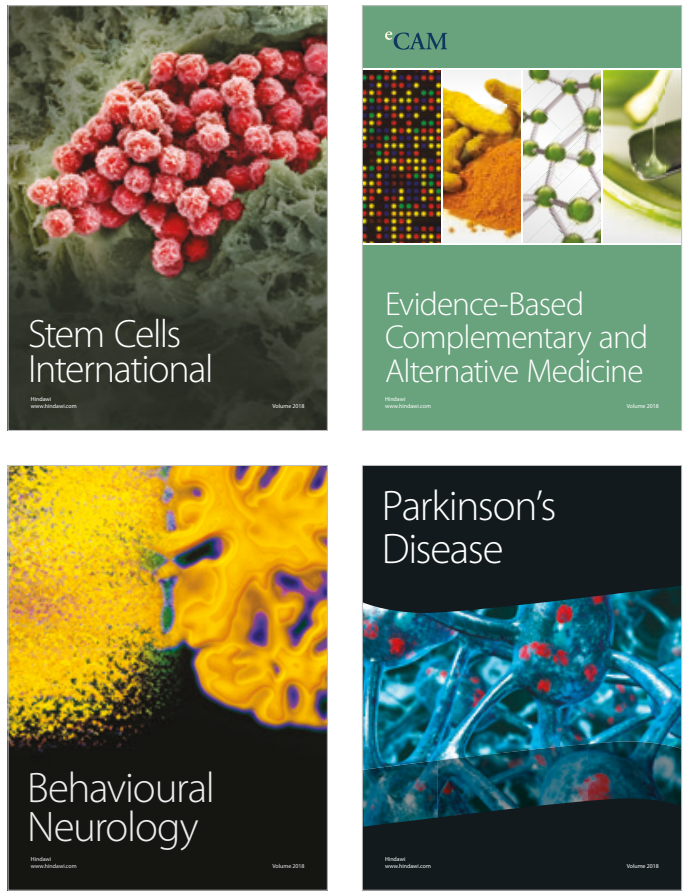

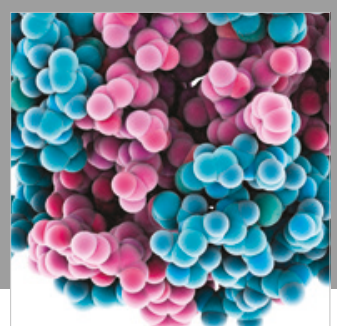

ournal of

Diabetes Research

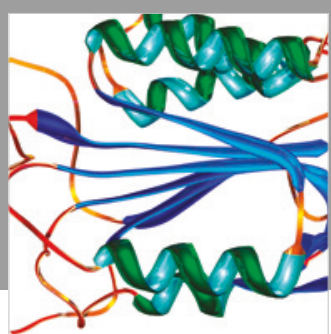

Disease Markers
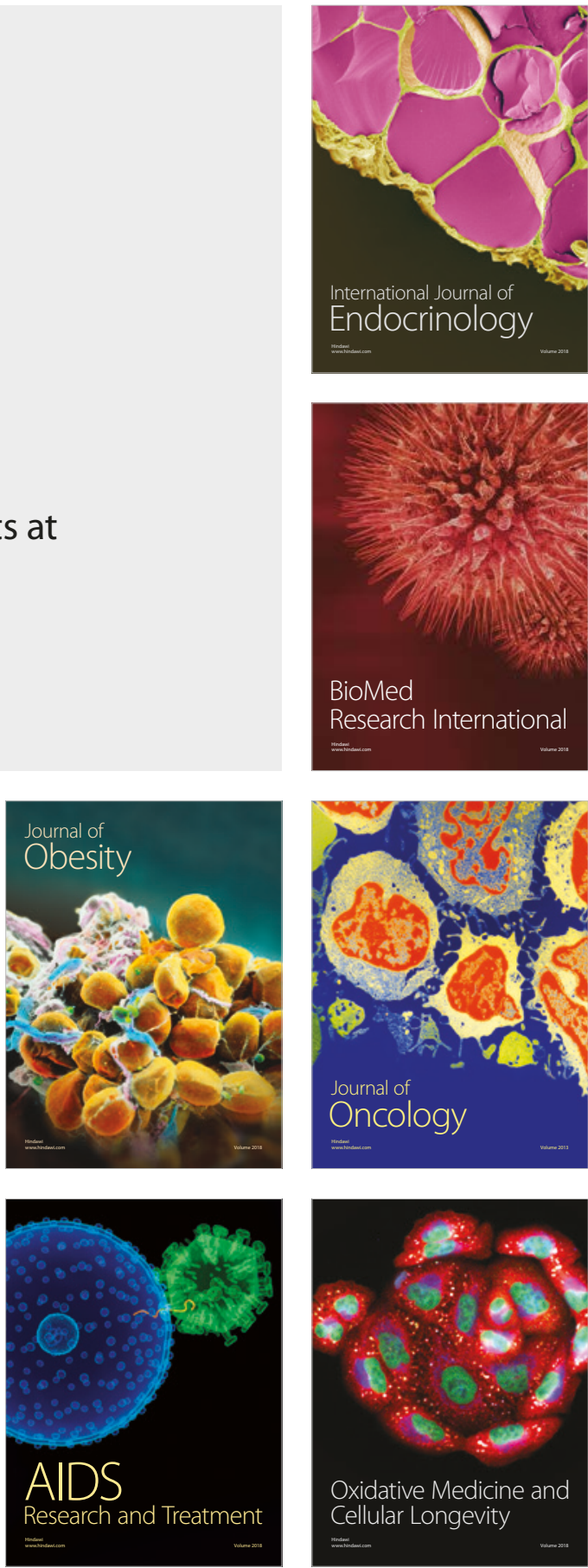